\title{
Dynamic phenotypes: illustrating a single-cell odyssey
}

\author{
William Wang $•$ Bijun Zhu $\cdot$ Xiangdong Wang
}

Received: 19 April 2017 /Revised: 28 April 2017 / Accepted: 29 May 2017 /Published online: 21 June 2017

(C) Springer Science+Business Media Dordrecht 2017

The importance of single-cell biology and systems biology is highlighted with the advanced technologies to understand cell behavior, heterogeneity, and evolution (Wang 2015; Qian et al. 2017). The "single-cell dynamic phenotypes" can be defined as time-dependent observable characteristics of single cells, e.g., morphology, biological properties, bio-behaviors, genetic changes, and productions. Single-cell dynamic phenotypes are used to define cancer pathological categories, progression, constraints, and mutations. Of these, single-cell sequencing plays a critical role in the measurement of intratumor heterogeneity which is closely associated with cancer genome instability, evolution, and drug resistance. Furthermore, biomarkers specific for drug efficacy and toxicity, molecular mechanisms of toxicology, and cell responses to targeted drugs are identified and validated using single-cell sequencing, systems biology, or models. Single-molecule imaging in single cells can trace intra-single-cell molecule expression, signal interactions, and locations. The multidimensional organization of genome function and mechanical phenotyping are also critical components of single-cell

\author{
W. Wang $(\bowtie) \cdot$ B. Zhu $\cdot$ X. Wang \\ Zhongshan Hospital Institute of Clinical Science, Fudan \\ University, Shanghai, China \\ e-mail: william_95a@hotmail.com \\ X. Wang \\ e-mail: xdwang@fuccb.com \\ W. Wang $\cdot$ B. Zhu $\cdot$ X. Wang \\ Shanghai Institute of Clinical Bioinformatics, Shanghai, China
}

biological functions. Stereoscopic single-cell biology becomes more important in the discovery and development of biomarkers (Niu et al. 2016; Wang and Song 2017). Thus, it is necessary to highlight the importance of single-cell dynamic phenotypes during various stages of evolution, microenvironmental changes, disease progression, and therapies.

Dynamic phenotypes of gene changes and mutations in single cells are dependent upon its microenvironmental contingencies, which drive the formation and complexity of intratumor heterogeneity and determine cell responses to drugs. Dynamic alterations of heredity and somatic genes contribute to the diversity of generations and mainly cause the initiation of cancer. For example, the immune-microenvironment contributes to the evolution of lung cancer heterogeneity through the certain immune cells and related cytokines (Wang et al. 2017). Dynamic heterogeneity during inherent or environmental changes plays an important role in the evolutionary framework of tumor development with large-scale genomic alterations. The association of clonal heterogeneity and tumor evolution that macro-evolutionary leaps involved in large-scale chromosomal alterations could drive tumor evolution and metastasis (McGranahan and Swanton 2017). Single-cell dynamic phenotypes change with microenvironmental alterations, resulting in drug resistance. While, targeted or non-targeted drugs per se also alter the microenvironment directly or indirectly, resulting in pharmacological effects, the occurrence of somatic gene 
mutations, and changes of cell susceptibility to therapy.

Variations of gene stability and regulation in cell development, transition, and DNA methylation can influence the appearance of single-cell dynamic phenotypes. Methylation maps for multiple cell types demonstrated spatial and temporal gene expression, cell identities, functional networks, and key lineage-specific regulators (Schultz et al. 2015). The methylation reporter can be generated by the minimal promoter of small nuclear ribonucleoprotein polypeptide $\mathrm{N}$ with the endogenous regions of differential methylation. Such process with green fluorescent protein in a sleeping transposon vector could facilitate the stable integration in the genome and visualize genomic methylation states at the single-cell resolution level (Stelzer et al. 2015). Cisregulatory element-acting signals and methylationsensitive promoters can present the severity of singlecell dynamic methylation of single cells. Endogenous methylation dynamics of non-coding regulatory elements and their correlation with transcription during cell-state transition and differentiation as well as reprogramming of somatic cells to pluripotency are monitored during many stages, including development, prior and post therapies, sensitivity and resistance, recovery, and progressiveness at the single-cell level.

Protein-based phenotypes of single cells are changeable and dynamic, especially in response to drugs. For example, cell heterogeneity can be described at multiple dimensions, levels, and omics. Protein profiles of single cells can be the most important characters of heterogeneity. With development of proteomic technologies, single-cell functional proteomics can be measured using a number of different protein analyses methods, e.g., microchip-based toolkits. The large-scale high-dimensional data from high-throughput single-cell proteomic assays demonstrate the functional heterogeneity and dynamics of immune cells, cell-cell interaction, highresolution snapshots of immune system functions, and phosphoprotein signaling networks ( $\mathrm{Lu}$ et al. 2017). This makes it possible to assess multiple molecular types and profiles in the same single-cell by multiomics, since gene sequencing and expression are only the part of single-cell profiling.

Simultaneous measurements of single-cell genomes, proteomes, epigenomes, and transcriptomes not only demonstrated the cell identity, phenotypes, expression, and variations but also the regulation, dynamics, interactions, and functions (Macaulay et al. 2017). Single- cell multiomics can provide a more stereoscopic picture of cell phenotypes and functions, which make the proposal and concept of an artificial intelligent cell possible (Niu et al. 2016). With development of clustered regularly interspaced short palindromic repeats (CRISPR), future single-cell genome engineering could result in a model created earlier than predicted (Fang and Wang 2016). The intelligent cells are also known as robot cells, whole-cell models, or smart cells and defined as a cell-like robot with learning capacity. The intelligent single cell can read and analyze omics-based measures, integrate and mine with their own databases and global resources, and provide a systemic illustration of complex cellular processes, dynamics, networks, and interactions of the synthetic circuits in a tested cell. The intelligent single cell can be used for target mechanism-based screening and validation of drug specificity and efficiency.

Single-cell surface protein profiles can describe cell phenotypes, while intracellular signal networks present protein-protein interactions and activations. These can be measured through different methods, e.g., flow and mass flow cytometry for membrane proteins, ELISpot for secreted proteins, image cytometry for membrane or intracellular proteins, cell arrays for intracellular protein, micro-droplets for membrane or intracellular proteins, micro-engraving for secreted or membrane proteins, or barcode chips for secreted, membrane, or cytoplasmic proteins (Wei et al. 2013; Tsioris et al. 2014). Several RNAs and proteins from a single human breast adenocarcinoma cell in one reaction chamber can be quantified using reverse transcriptase (Genshaft et al. 2016). Such integrated protocol provides the potential to simultaneously profile transcriptomic and proteomic responses of single cells to a chemical perturbation.

Dynamics of single-cell phenotypic heterogeneity can be used to track responses of individual cell changes to drugs. In order to detect the heterogeneity in the morphological phenotype response to chemical perturbations, and microenvironmental alterations, the singlecell dynamic phenotypes of heterogeneous populations were measured by live cell imaging and fluorescence time-lapse microscopy (Patsch et al. 2016). The value of single-cell dynamic phenotypes is highly dependent upon the protocol of the study design and questions to be answered. High-throughput imaging platforms serve as a powerful tool to dynamically monitor cell proliferation, differentiation, mobility, phosphorylation, and response to therapeutic perturbation. Zhang et al. 
(2012) applied such platforms with an interventional strategy of different inhibitors and found interleukin-8 production from lung cancer cells through the EGFEGFR-PI3K-Atk-Erk pathway. The high-throughput image platform includes image acquisition, phenotype tracking, data filtering and analyses, and integrative data mining with cell features and digital assays. With the platform, a large number of biological effects are investigated, including phosphoinositide 3-kinase in airway epithelial remodeling, soluble fibrinogen-like protein 2 in transplanted cell survival, pyrroloquinoline in cancer cell apoptosis via the mitochondrial-dependent pathway, adrenaline in proliferation and migration of mesenchymal stem cells, keratinocyte growth factor- 2 in airway epithelial cell injury, and EGF-EGFR signaling pathway in hepatocellular carcinoma (Fang et al. 2013; Wang et al. 2014; Min et al. 2014; Wu et al. 2014; Fang et al. 2014; Huang et al. 2014). The high-throughput imaging system was recently used to explore the regulatory mechanisms of TGF- $\beta 1$-induced fibrogenesis of human alveolar epithelial cells and the inhibitory effects of drugs in hepatocellular carcinoma progression (Shi et al. 2016; Fang et al. 2016). Although those studies observed phenotypic alterations of the same type individual cells, data filtering and analyses were not performed and presented on single cells. Patsch et al. (2016) developed the new strategy and protocol to investigate phenotypes of single cells in the highthroughput imaging system in a different aspect of gene- and protein-based phenotype of single cells.

Dynamic genotypes and heterogeneity of single cells demonstrate perturbation and mutations by measuring single-cell RNA and transcriptomics. Single-cell RNA sequencing (scRNA-seq) is used to identify transcript start sites, novel transcripts, splicing variants, transcript isoforms, candidate biomarkers, low-copy number transcripts, and siRNA screen. Liu et al. (2016) measured long non-coding RNAs (lncRNAs) profiles of single cells from polyadenylated and total RNA from human neocortex at different stages of development, using strand-specific RNA-seq. The lncRNAs of neocortex single cells appear cell type-specific and demonstrate the heterogeneity among cells. Cellular lncRNAs are hardly detected and quantified low and diverse concentrations of IncRNAs in tissues. scRNA-seq can be applied for the identification of driver genes in molecular diseases. For example, elevated levels of an lncRNA LOC646329 in single radial glia cells were observed which in contrast were hardly observed at the tissue level (Liu et al. 2016). scRNA-seq has more value in the discovery and identification of dynamic phenotypes among subgroups of cells within tissues, driver elements of transcriptome, and cell type- or function-specific genes. Kim et al. (2015) measured gene expression and mutation profiling, as well as single-nucleotide variations of lung cancer patient-derived xenograft tumor cells using scRNA-seq to understand intratumor genetic and functional heterogeneity and find transcriptome signatures of anti-cancer drug treatments. Different from pathological and clinical categories of diseases, tumor cells will be newly categorized into subgroups associated with anti-cancer drug resistance, according to single-cell genotypes. Gene-based new categories of tumor cells can facilitate and optimize the development of clinical anti-cancer strategies.

The measurement of single-cell dynamic phenotypes can be combined with other methodological strategies and technologies to explore molecular mechanisms of cell originated functions and evolution. For example, the development and heterogeneity and molecular trajectories of $\mathrm{T}$ helper (Th) subsets Th1 and Tfh cells during infection with P. chabaudi chabaudi AS, a rodent-infective strains of malaria, were monitored with a computational modeling strategy (a mixture of Gaussian processes models) with scRNA-seq (Lönnberg et al. 2017). Those important findings demonstrated that splenic monocytes/macrophages, dendritic cells, and other myeloid cells could support the Th1 fate by influencing bifurcation during infection. Of the chemokines, CXCR5 was the only chemokine receptor that was significantly associated with bifurcation through cellular interaction with B-cell follicles, chemoattractant signals from different zones of the spleen, and intercellular communication as a major driver gene. In addition, the single-cell dynamic phenotype plays critical roles in target-based drug discovery and development (Heath et al. 2016) and understanding of systems heterogeneity (Wang and Wang 2017). System heterogeneity among single cells has been suggested as a critical merging topic in the discovery and development of diagnostic biomarkers and therapeutic targets. A recent study investigated genetic heterogeneity and temporal fluctuations of cellular phenotypes, e.g., growth-related proteins in single cells, by measuring the fitness landscapes of arbitrary phenotypic traits in the form of population lineage trees on the basis of 


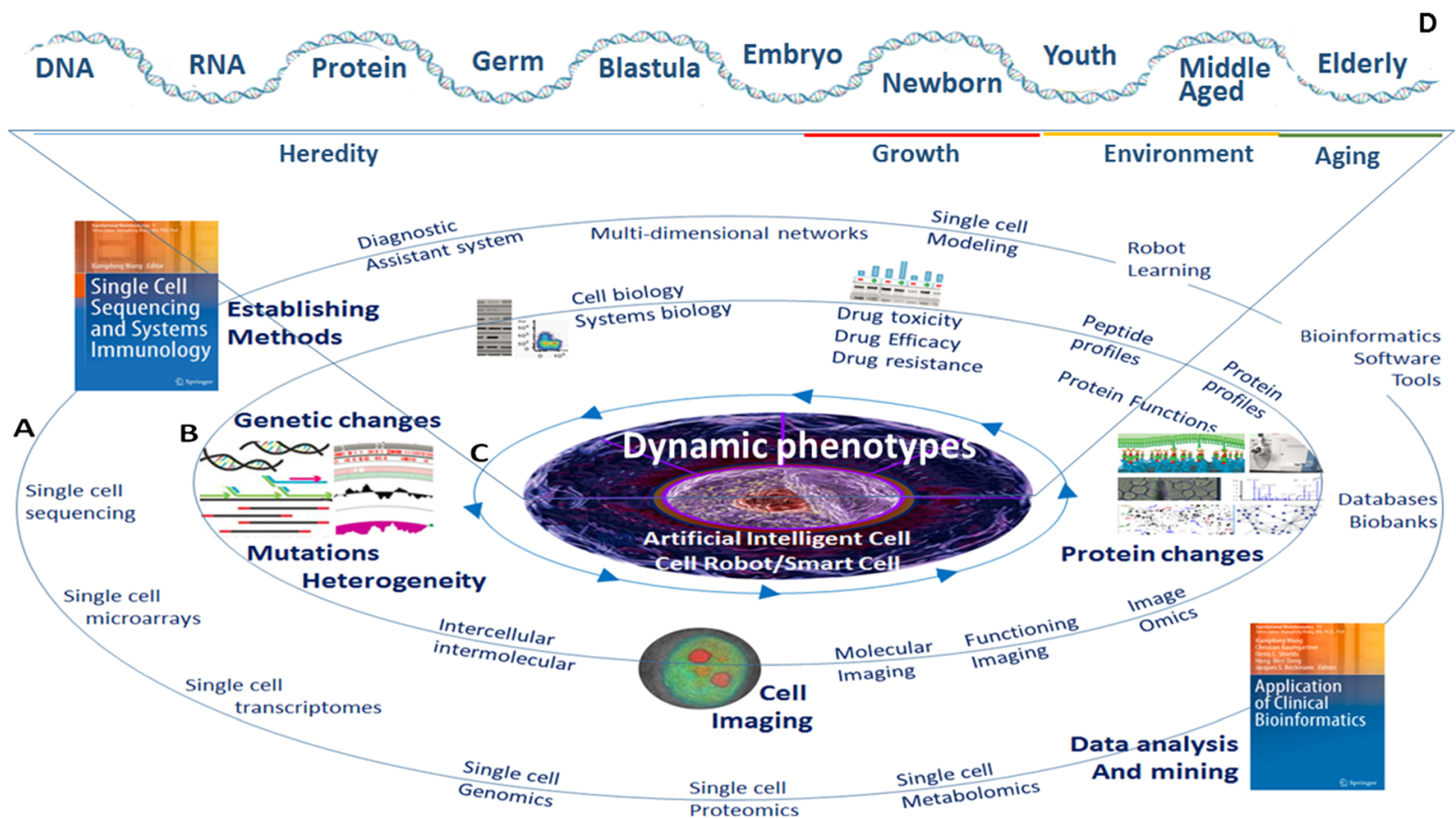

Fig. 1 The principle and importance of single-cell dynamic phenotypes to understand the evolution and progression of aging and diseases. A circle: a large number of methodologies are discovered and developed to measure single-cell gene expression, sequencing, transcriptional networks, protein profiles, and metabolomics. Data generated from those measures can be stored and mined by the integration with global databases. Those multidimensional omics and their big data can be used to establish an intelligent cell. $B$ circle: single-cell dynamic phenotypes are used to understand gene mutations, heterogeneity, morphological characters, protein functions, responses to drugs, and systems biology. $C$ circle: an artificial intelligent cell also named cell robot, smart cell, or cell model can be created on basis of dynamic phenotypes and multiomics. The intelligent cell has the capacity of reading, learning, analyzing, mining, and reporting to monitor dynamic phenotypes of cells, organs, and human from DNA to embryo and to the elder $(D)$. Single-cell dynamic phenotypes are altered during the evolution, differentiation, and progression of cells due to multiple influencing factors

single-cell phenotypes are regulated at multiple levels by hereditary or somatic factors, e.g., epigenetics, chromatin stability, or external challenges. Single-cell dynamic phenotypes can demonstrate more about the contributions of tumor heterogeneity and evolution to carcinogenicity, metastasis, and responses to targeted therapies. Thus, dynamic phenotypes at both the gene and protein levels make it possible to have new therapeutic strategies, illustrate a single-cell odyssey and develop an artificial intelligent cell with the capacity of learning and analyzing.

\section{References}

Fang H, Wang W. Could CRISPR be the solution for gene editing's Gordian knot? Cell Biol Toxicol. 2016;32:465.

Fang X, Li K, Tao X, Chen C, Wang X, Wang L, et al. Effects of phosphoinositide 3-kinase on protease-induced acute and 
chronic lung inflammation, remodeling, and emphysema in rats. Chest. 2013;143(4):1025-35.

Fang X, Wang L, Shi L, Chen C, Wang Q, Bai C, et al. Protective effects of keratinocyte growth factor- 2 on ischemiareperfusion-induced lung injury in rats. Am J Respir Cell Mol Biol. 2014;50(6):1156-65.

Fang T, Hou J, He M, Wang L, Zheng M, Wang XD, et al. Actinidia chinensis Planch root extract (acRoots) inhibits hepatocellular carcinoma progression by inhibiting EP3 expression. Cell Biol Toxicol. 2016;32(6):499-511.

Genshaft AS, Li S, Gallant CJ, Prakadan SM, Ziegler CGK, Lundberg $\mathrm{M}$, et al. Multiplexed, targeted profiling of singlecell proteomes and transcriptomes in a single reaction. Genome Biol. 2016;17:188.

Heath JR, Ribas A, Mischel PS. Single cell analytic tools for drug discovery and development. Nat Rev Drug Discov. 2016;15(3):204-16.

Huang P, Xu X, Wang L, Zhu B, Wang XD, Xia J. The role of EGF-EGFR signalling pathway in hepatocellular carcinoma inflammatory microenvironment. J Cell Mol Med. 2014;18(2):218-30.

Kim KT, Lee HW, Lee HO, Kim SC, Seo YJ, Chung W, et al. Single-cell mRNA sequencing identifies subclonal heterogeneity in anti-cancer drug responses of lung adenocarcinoma cells. Genome Biol. 2015;16(1):127.

Liu SJ, Nowakowski TJ, Pollen AA, Lui JH, Horlbeck MA, Attenello FJ, et al. Single-cell analysis of long non-coding RNAs in the developing human neocortex. Genome Biol. 2016;17:67.

Lönnberg T, Svensson V, James KR, Fernandez-Ruiz D, Sebina I, Montandon R, et al. Single-cell RNA-seq and computational analysis using temporal mixture modelling resolves Th1/Tfh fate bifurcation in malaria. Sci Immunol. 2017;2(9): eaal2192.

Lu Y, Yang L, Wei W, Shi Q. Microchip-based single-cell functional proteomics for biomedical applications. Lab Chip. 2017;17(7):1250-63. doi:10.1039/c7lc00037e.

Macaulay IC, Ponting CP, Voet T. Single-cell multiomics: multiple measurements from single cells. Trends Genet. 2017;33(2): 155-68.

McGranahan N, Swanton C. Clonal heterogeneity and tumor evolution: past, present, and the future. Cell. 2017;168(4): 613-28.

Min Z, Wang L, Jin J, Wang XD, Zhu B, Chen H, et al. Pyrroloquinoline quinone induces cancer cell apoptosis via mitochondrial-dependent pathway and down-regulating cellular Bcl-2 protein expression. J Cancer. 2014;5(7):609-24.

Niu F, Wang DC, Lu J, Wu W, Wang X. Potentials of single-cell biology in identification and validation of disease biomarkers. J Cell Mol Med. 2016;20(9):1789-95.
Nozoe T, Kussell E, Wakamoto Y. Inferring fitness landscapes and selection on phenotypic states from single-cell genealogical data. PLoS Genet. 2017;13(3):e1006653.

Patsch K, Chiu CL, Engeln M, Agus DB, Mallick P, Mumenthaler SM, et al. Single cell dynamic phenotyping. Sci Rep. 2016;6: 34785 .

Qian M, Wang DC, Chen H, Cheng Y. Detection of single cell heterogeneity in cancer. Semin Cell Dev Biol. 2017;64:1439.

Schultz MD, He Y, Whitaker JW, Hariharan M, Mukamel EA, Leung D, et al. Human body epigenome maps reveal noncanonical DNA methylation variation. Nature. 2015;523:2126.

Shi L, Dong N, Fang X, Wang X. Regulatory mechanisms of TGF- $\beta 1$-induced fibrogenesis of human alveolar epithelial cells. J Cell Mol Med. 2016;20(11):2183-93.

Stelzer Y, Shivalila CS, Soldner F, Markoulaki S, Jaenisch R. Tracing dynamic changes of DNA methylation at singlecell resolution. Cell. 2015;163(1):218-29.

Tsioris K, Torres AJ, Douce TB, Love JC. A new toolbox for assessing single cells. Annu Rev Chem Biomol Eng. 2014;5: 455-77.

Wang XD. In: Wang XD, editor. Single cell sequencing and systems immunology, Serial books: translational bioinformatics, vol. 5. Netherlands: Springer; 2015. doi:10.1007 1978-94-017-9753-5.

Wang J, Song Y. Single cell sequencing: a distinct new field. Clin Transl Med. 2017;6:10.

Wang DC, Wang X. Systems heterogeneity: an integrative way to understand cancer heterogeneity. Semin Cell Dev Biol. 2017;64:1-4.

Wang L, Yang C, Xu M, Hu M, Wang XD, Zhu T. The role of soluble fibrinogen-like protein 2 in transplantation: protection or damage. Transplantation. 2014;97(12):1201-6.

Wang L, Zhu B, Zhang M, Wang X. Roles of immune microenvironment heterogeneity in therapy-associated biomarkers in lung cancer. Semin Cell Dev Biol. 2017;64:90-7.

Wei W, Shin YS, Ma C, Wang J, Elitas M, Fan R, et al. Microchip platforms for multiplex single-cell functional proteomics with applications to immunology and cancer research. Genome med. 2013;5(8):75.

Wu X, Wang Z, Qian M, Wang L, Bai C, Wang XD. Adrenaline stimulates the proliferation and migration of mesenchymal stem cells towards the LPS-induced lung injury. J Cell Mol med. 2014;18(8):1612-22.

Zhang Y, Wang LY, Zhang MM, Jin ML, Wang X. Potential mechanism of interleukin-8 production from lung cancer cells: an involvement of EGF-EGFR-PI3K-Atk-Erk pathway. J Cell Physiol. 2012;227(1):35-43. 